TEE guidance. TEE may also be beneficial for reducing the risk of paraplegia.

Although the priority of $\mathrm{CT}$ assessment in acute aortic dissection is not in dispute, TEE may be a useful adjunct to overcome its limitations.

\section{Reference}

1. Shiga T, Wajima Z, Apfel CC, Inoue T, Ohe Y. Diagnostic accuracy of transesophageal echocardiography, helical computed tomography, and magnetic resonance imaging for suspected thoracic aortic dissection: systematic review and meta-analysis. Arch Intern Med. 2006;166:1350-6.

\title{
Aortoesophageal fistula associated with a Kommerell diverticulum and right-sided aortic arch
}

\author{
Karl G. Reyes, MD, ${ }^{\mathrm{a}}$ Inderjit S. Gill, MD, ${ }^{\mathrm{a}}$ Thomas Temes, MD, ${ }^{\mathrm{a}}$ and Noreen C. Griffin, CNP, ${ }^{\mathrm{b}}$ Cleveland, Ohio
}

Aortoesophageal fistula (AEF) is rare and often fatal complication of vehicular trauma. In the past, it has also been reported in the presence of congenital arch anomalies. We report here a case of traumatic AEF in a patient with both a right-sided aortic arch and a Kommerell diverticulum, the first such in the literature, as well as its successful management by staged open surgical repair.

\section{CLINICAL SUMMARY}

A 26-year-old man was taken to the emergency department after a vehicular crash in which he had been the ejected, unrestrained driver. Injuries noted on admission were primarily intracranial, with a depressed mental status necessitating mechanical ventilation. The patient's mental status eventually improved to the point at which he could be weaned from the ventilator and required only a percutaneous endoscopic gastrostomy tube for feeding. He was discharged to a rehabilitation center 3 weeks later.

During the patient's stay at the rehabilitation center, approximately 4 weeks after the crash, he had massive gastrointestinal bleeding, with bright red blood coming out of his percutaneous endoscopic gastrostomy tube. The patient was taken to a nearby hospital, where he was resuscitated and subsequently taken to the endoscopy suite. On esophagogastroscopy, large amounts of blood and blood clots were seen, but with no identifiable source of bleeding. Because of the suddenness of the event, the diagnosis of AEF was considered, and the patient was flown to our institution. On arrival, the patient had no active bleeding. Diagnostic studies included chest radiography, results of which were unremark-

\footnotetext{
From the Department of Thoracic and Cardiovascular Surgery, The Cleveland Clinic Foundation, ${ }^{\mathrm{a}}$ and the Metrohealth Medical Center, ${ }^{\mathrm{b}}$ Cleveland, Ohio. Received for publication Nov 15, 2007; accepted for publication Nov 25, 2007.

Address for reprints: Karl G. Reyes, MD, Department of Thoracic and Cardiovascular Surgery, The Cleveland Clinic Foundation, 9500 Euclid Ave H35, Cleveland, OH 44195 (E-mail: reyesk2@ccf.org).

J Thorac Cardiovasc Surg 2008;136:1091-3

$0022-5223 / \$ 34.00$

Copyright $(C) 2008$ by The American Association for Thoracic Surgery doi:10.1016/j.jtcvs.2007.11.057
}

able except for findings suggestive of a right-sided aortic arch. Chest CT scan (Figure 1) confirmed the presence of a right-sided aortic arch and descent of the aorta on the right side of the chest. In addition, there was an aberrant left subclavian artery with an aneurysmal origin, consistent with a Kommerell diverticulum, and an anteriorly displaced esophagus that narrowed at the level of the aberrant left subclavian artery. The suspected AEF, however, was not seen on the CT scan. Esophagogastroscopy was repeated and this time showed a nonbleeding, pulsating, $0.7-\mathrm{cm}$ ulcer within the esophagus at $25 \mathrm{~cm}$, corresponding to the level of the aortic knuckle. The patient was then taken to the operating room.

En route to the operating room, the patient again had massive bleeding. A standard left posterolateral thoracotomy through the fourth interspace was done. The posterior mediastinum was exposed, and the preexisting congenital anomalies were identified. The esophagus was then mobilized until an area of adherence between the esophagus and aorta was found, at the level of the distal aortic arch and very close to the Kommerell diverticulum. Pledgeted sutures were then passed and tied down in this area. Intraoperative esophagoscopy confirmed the cessation of bleeding. At this point, although hemostasis had been achieved, the patient had hypothermia and severe acidosis. It was therefore decided that the esophageal injury would be managed later, and the patient was taken to the intensive care unit. The patient's postoperative course went remarkably well, and he was returned to the operating room on the 6th postoperative day. Before reopening of the thoracotomy, esophagogastroscopy was performed at the operating room, and the previously identified lesion was once again seen, as was pledget material that appeared to be present within the lesion. The left posterolateral thoracotomy was subsequently reopened. The esophagus was mobilized, and the fistula was excised with a small disk of esophagus left attached. The esophageal defect was then repaired primarily, and a nasogastric tube was inserted in position adjacent to the repair. On the aortic side, the polytetrafluoroethylene pledgets were removed and 


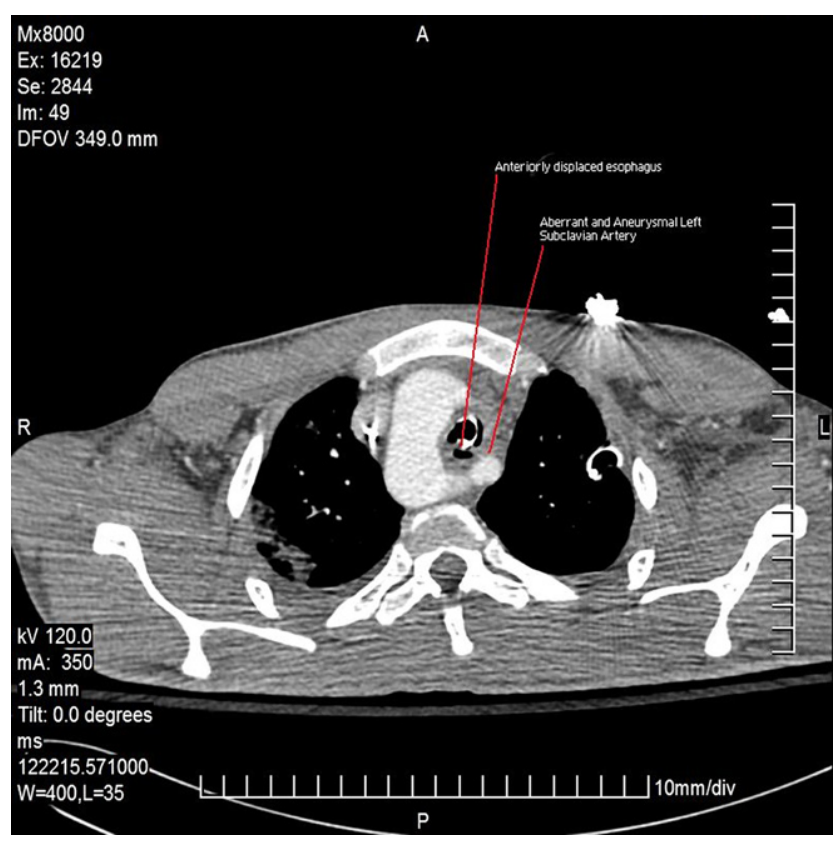

FIGURE 1. CT scan demonstrating right-sided aortic arch and esophagus narrowed anteriorly at level of aberrant subclavian artery. Kommerell diverticulum is present and can be seen adjacent to left subclavian artery.

replaced with autologous pericardial pledgets to decrease the risk of potential infection. A third intercostal muscle flap was then interposed between the repairs. This concluded the second stage of the operation.

The patient's postoperative course was unremarkable. He was extubated the day after his second operation. His mental status remained essentially unchanged from admission, and 11 days after the second operation, he was discharged to the rehabilitation facility.

The patient was seen again 10 days later for outpatient follow-up. He appeared to have recovered well from his surgery, and another CT scan showed no evidence of disruption of either repair. Subsequent follow-up calls during the next couple of months yielded positive reports, with the patient continuing to recover neurologically. His percutaneous endoscopic gastrostomy tube was eventually removed, and he was eventually able to tolerate a normal diet. There have been no further episodes of gastrointestinal bleeding. It is now 5 years since the crash. The patient is alive and well, fully functional, and seeking employment.

\section{DISCUSSION}

AEF was first described by Dubrueil ${ }^{1}$ in 1818 after a soldier swallowed a piece of rib, which perforated his descending thoracic aorta and eventually caused his death. Since then, there have been only a few reported cases in the literature of AEF secondary to trauma, and only 1 other in a patient with a right-sided aortic arch. ${ }^{2}$ Most AEFs reported in the literature have typically been related to ruptured thoracic

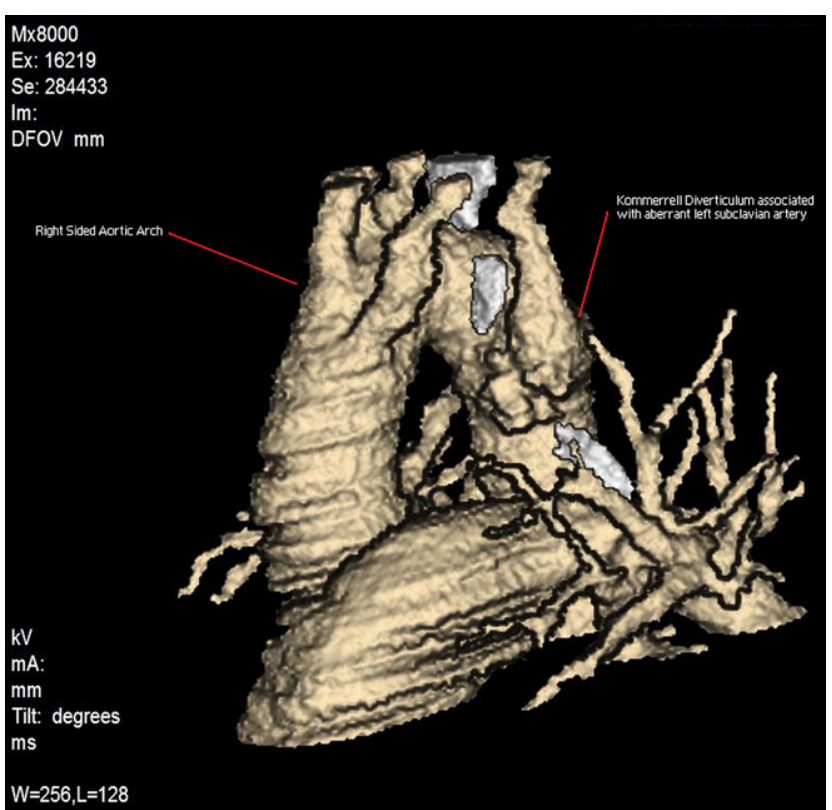

FIGURE 2. Three-dimensional reconstruction of aortic arch.

aneurysms. Hollander and colleagues ${ }^{3}$ described 500 cases of AEF, and descending thoracic aneurysms accounted for $51 \%$ of the cases. Other causes included foreign-body perforations, thoracic malignancies, and postoperative complications; these made up approximately $40 \%$ of the cases. The remaining described cases of AEF were caused by esophageal reflux, tuberculosis, corrosive esophagitis, congenital anomalies, atherosclerotic disease, or instrumentation. There were only 6 reported cases $(1 \%)$ of traumatic AEF in Hollander and colleagues' series. ${ }^{3}$

The diagnosis of AEF is often made at clinical presentation. Survival is rare, because most patients die immediately. If the patient survives the sentinel bleeding, diagnostic evaluation should follow immediately. Emergency endoscopy is often the initial diagnostic test. In stable patients, other diagnostic tests, such as CT scan and aortography, are reasonable for confirmation. In unstable patients, immediate surgical management is the only option. The tenets of successful surgical treatment are control of hemorrhage with repair of the aortic injury and individualized management of the esophagus, depending on patient stability and the underlying esophageal pathologic condition. The injury to the esophagus varies, from a simple, small perforation that requires primary repair to a completely destroyed esophagus with a contaminated thorax and mediastinum that requires esophagectomy and staged reconstruction. Any suture lines mandate interposition flaps. Broad-spectrum antibiotics are used to control infection resulting from esophageal leakage into the chest and mediastinum.

There has been only 1 other case reported in the literature of AEF in a patient with a congenital right-sided aortic arch, 
and no previous reports of AEF in a patient with both a rightsided aortic arch and Kommerell diverticulum. Kommerell diverticulum (Figure 2) is a saccular aneurysmal dilation at the origin of an aberrant right or left subclavian artery. It is a rare anomaly that occurs in association with a double or left aortic arch and an aberrant right subclavian artery $(0.5 \%$ of the population) or with a right aortic arch and an aberrant left subclavian artery $(0.1 \%)$. Embryologically, it represents the persistent distal end of the interrupted fourth aortic arch, between the carotid and subclavian arteries. ${ }^{4}$ The question arises as to whether this congenital anomaly increases the likelihood of development of an AEF in response to disease or disruption. Kommerell diverticulum represents a likely area of weakening in the aortic wall that can also compress the esophagus and, in theory, could cause erosion of the structure into each other, especially when disruption of adventitial layers has occurred, such as with trauma.
In summary, long-term survival can be achieved with early diagnosis and prompt surgical management of AEF. Because this condition presents in a catastrophic manner, younger and healthier patients are more likely to have better outcomes. In our case, the patient's young age and the relatively small size of his AEF, as well as prompt diagnosis and surgical treatment, proved the ultimate determinants of success and are why he remains alive to this day.

\section{References}

1. Dubreuil O. Observations sur la perforation de l'esopphage et de l'aorte thoracique par une portion d'os avale: avee de reflexions. J Univ Sci Med. 1818;9:357-63.

2. Rodriguez E, Park P, Cowan S, Weinstein M, Kaulback K, Friedberg JS, et al Complicated management of a traumatic aortoesophageal fistula in a patient with a right-sided aortic arch. J Trauma. 2004;56:691-3.

3. Hollander JE, Quick G. Aortoesophageal fistula: a comprehensive review of the literature. Am J Med. 1991;91:279-87.

4. Arciniegas E, Hakimi M, Hertzler JH, Farooki ZQ, Green EW. Surgical management of congenital vascular rings. J Thorac Cardiovasc Surg. 1979;77:721-7.

\section{Recurrent sarcoma originating from the pulmonary artery 6 years after extensive thoracic resection}

Christine Fegbeutel, MD, ${ }^{a}$ Martin Strüber, MD, ${ }^{a}$ Jan U. Becker, MD, ${ }^{b}$ Florian Länger, MD, ${ }^{\mathrm{b}}$ Nicolas Dickgreber, MD,

Axel Haverich, MD, ${ }^{a}$ and Stefan Fischer, MD, MSc, ${ }^{a}$ Hannover, Germany

Sarcoma originating from the pulmonary artery (PA) is a rare tumor. In 1923, Mandelstamm ${ }^{1}$ was the first to describe this disease in an autopsy. Since then, fewer than 200 cases overall have been reported. Because of its rarity and insidious growth characteristics, PA sarcoma is often mistaken for pulmonary embolism, leading to inappropriate therapy such as prolonged anticoagulation or thrombolysis. ${ }^{2}$

Prognosis is usually poor with a median survival of approximately 12 months with surgical resection of the tumor and 1.5 months without surgical resection of the tumor. Several reports have shown that chemotherapy and radiotherapy may lead to prolonged survival. Only a few cases of complete remission after radical surgical resection have been re-

\footnotetext{
From the Department of Cardiac, Thoracic, Transplant, and Vascular Surgery, ${ }^{\mathrm{a}}$ Department of Pathology, ${ }^{b}$ and Department of Respiratory Medicine, ${ }^{\mathrm{c}}$ Hannover Medical School, Hannover, Germany.

Received for publication Nov 21, 2007; accepted for publication Feb 5, 2008.

Address for reprints: Stefan Fischer, MD, MSc, Director, Hannover Thoracic Surgery and Lung Support Program Department of Cardiac, Thoracic, Transplant, and Vascular Surgery, Hannover Medical School, Carl-Neuberg-Strasse 1, 30625 Hannover, Germany (E-mail: fischer.stefan@mh-hannover.de).

J Thorac Cardiovasc Surg 2008;136:1093-5

$0022-5223 / \$ 34.00$

Copyright (c) 2008 by The American Association for Thoracic Surgery

doi:10.1016/j.jtcvs.2008.02.006
}

ported. The longest survival times reported are 3.5 years and 5.5 years. ${ }^{3,4}$

We report the case of a 48-year-old female patient with recurrent sarcoma of the main PA mimicking fulminant PA embolism 6 years after extensive resection of the primary tumor.

\section{CLINICAL SUMMARY}

A previously healthy 42-year-old patient developed progressive respiratory distress over a period of several weeks as the result of pleomorphic sarcoma of the mediastinum with infiltration of the left atrium, both pulmonary arteries, the superior vena cava, right main stem bronchus with total occlusion of the right upper lobe, and lung parenchymal infiltration. After initial chemotherapy, surgical resection was performed, including right pneumectomy, resection of the PA bifurcation, and partial resection of the left atrium. The left atrium was patched with pericardial tissue, and the PA was reestablished in an end-to-end technique.

Because of perioperative acute pulmonary embolism, oral anticoagulation was initiated. The patient recovered with 